\title{
Groundwater Possibility Detection using Swarm Intelligence Technique
}

\author{
RavinderKaur \\ Computer Science Engineering \\ IETBhaddal Technical Campus \\ City:Roopnagar, Country:India \\ Dr.Sanjay Singla \\ Computer Science Engineering \\ IET Bhaddal Technical Campus \\ City:Rupnagar, Country:India
}

\begin{abstract}
From the ancient years, natural social species fascinated the individual and researchers for the seamless intelligence work solutions. Swarm intelligence is also a nature inspired computing technique that is inspired from the social species like small ants, termites, wasp, bee etc. There are various techniques of swarm intelligence. In this work, we are using swarm intelligence based Biogeography Based Optimization (BBO) for the groundwater possibility detection. Here, $\mathrm{BBO}$ is integrated with Case Based Reasoning to extract the available cases for the research solution of groundwater possibility estimation. For experimentation, we have considered an expert dataset with feature of slope, landuse, landform, geology, soil type and lineament. Groundwater possibility detection is considered to explore the possibility with the given attributes for any exploration area. The proposed concept is elaborated with research solution.
\end{abstract}

Keywords- Biogeography Based Optimization, Case Based Reasoning, Ground Water Possibility, Swarm Intelligence

\section{INTRODUCTION}

Swarm Intelligence is an artificial concept inspired from the collaborative multiagent social species that work intellectually to design intelligent system and optimized algorithm. There is the existence of various social species like termites, wasp, bee, flying birds, school of fishes that work as a collaborative behavior [1]. The global cooperation of these social species to work collaboratively is due to strategy of self organization. Self organization is a dynamic strategy where agents create global system by interaction of local level components. Based on the variety of social species of insects and animals, there exists a number of Swarm Intelligence techniques. Some of the popular swarm intelligence techniques are listed Bat Algorithm, Ant Colony Optimization, Cuckoo Search, Firefly Algorithm, Intelligent Water Drops Algorithm, Biogeography Based Optimization, Particle Swarm Optimization and Artificial Bee Colony Optimization [2].

From the list of swarm concepts, we are considering Biogeography Based Optimization which is a swarm based algorithm that is inspired by the migrating behaviour of the species as per the ecosystem. The natural process of migration of species from one island to another according to the survival circumstances is adapted for the generation of this algorithm. In BBO, the islands are considered as the solution of the problem \& migration behaviour is taken as sharing of feature solutions [3]. This natural phenomenon can be used for the solution of various hard computational problems.

In this research work, we are using BBO with CBR for groundwater possibility detection.CBR uses previously experience based knowledge instead of solely dependent on problem domain, their description and available resources. The previous experienced based knowledge is considered as the cases for the problem solution. These cases are considered as the iterations to solve the problem. In CBR System, there is a problem solving life cycle which is a structure of four parts: Retrieve, Reuse, Revise and Retain [4].

For experimentation, we are using expert dataset having six attributes of slope, landuse, landform, geology, soil type and lineament to detect the possibility of groundwater. There are different combinations of these six attributes to show possibility as per feature match found. 
Other sections of the paper are described as: Section II presents the related work of groundwater possibility detection, Section III explains the methodology of the proposed concept with its work flow and Section IV concludes the paper.

\section{RELATED WORK}

This section describes the work related to groundwater possibility detection using different concepts.

\section{A. Groundwater Possibility}

Panchal et al. (2009) [5] has introduced the concept of swarm based wave of swarms with case based reasoning for the groundwater possibility detection. Authors have used the expert dataset with geological features of Geology, Landform, Soil Type, Land Use, Lineament, and Slope. Results are evaluated in the form of possibility factor of low, moderate and high. Further Panchal et al. (2013) [6] has extended the work for the same dataset by using case based reasoning and cuckoo search algorithm. The main assumption considered for this low, moderate and high concept is existence of host nest iteration as per cuckoo bird. For the considered cases of cuckoo nest with case based author claims to achieve a great level of accuracy as compare to wave of swarm method. Then, Gupta et al. (2014) [7]used the same dataset and implemented using robotic perception and autonomic computing. The data used for perception is stored in the microprocessors. Further the results are calculated in the form of depth of the groundwater level. This concept is prepared to train a robot for the detection of groundwater depth without digging the ground surface.

\section{B. Swarm Intelligence}

Kennedy and Eberhart (1995) [8] developed the Particle Swarm Optimization algorithm. It is swarm based algorithm developed by the inspiration of social working behaviour of flying birds. Dorigo et al. (1996) [9] developed the Ant Colony Optimization algorithm. It is also a swarm based algorithm, inspired by the working behaviour of the small ants. In ACO, small ants have the feature to leave a footmark for the ant to follow it. This footmark print is a substance known as Pheromone. D. Karaboga (2005) [10] developed the Artificial Bee Colony Optimization algorithm. It is swarm based optimization algorithm and developed by the inspiration of the working behaviour of honey bees. D. Simon (2008) [11] developed a nature inspired Biogeography Based Optimization algorithm. It is also a swarm based algorithm and was developed to solve the optimization problems. It is inspired by the species who change their living home as per the environmental conditions. These environmental conditions are known as Habitat Suitability Index (HSI). Yang and Deb (2009) [12] developed the Cuckoo Search algorithm. It is nature-inspired algorithm based on the working behaviour of the cuckoo bird to store their egg into other's nest. Cuckoo bird is so clever that it stores their egg into some other's nest. Kari and Rozenberg (2010) [13] reviews the many facets of Natural Computing such as Cuckoo Search, Particle Swarm Optimization, Biogeography Based Optimization, Bee Colony Optimization, Cellular Automata, Intelligent Water Drop, Membrane Computing, Genetic Algorithm, Neural Network, and Firefly Algorithm.

\section{RESEARCH METHODOLOGY}

The proposed concept is conducted in MATLAB 8.3.0.532 with Windows 7 based system having 4GB of RAM, 500GB of HDD, a dual core Intel(R) Core(TM) i7 CPU. Expert dataset is considered in the form of input attributes of slope, landuse, landform, geology, soil type and lineament and output results will be evaluated in the form of low, moderate and high possibility of groundwater. Here, we are using integrated concept of BBO and CBR for groundwater exploration. Initially, use cases are created using the expert dataset which are actually the habitat suitable condition for BBO species. After the number of iterations, we can get the possibility results of low, moderate or higher. Steps for the research methodology are described below with flowchart as shown in figure 1 . 


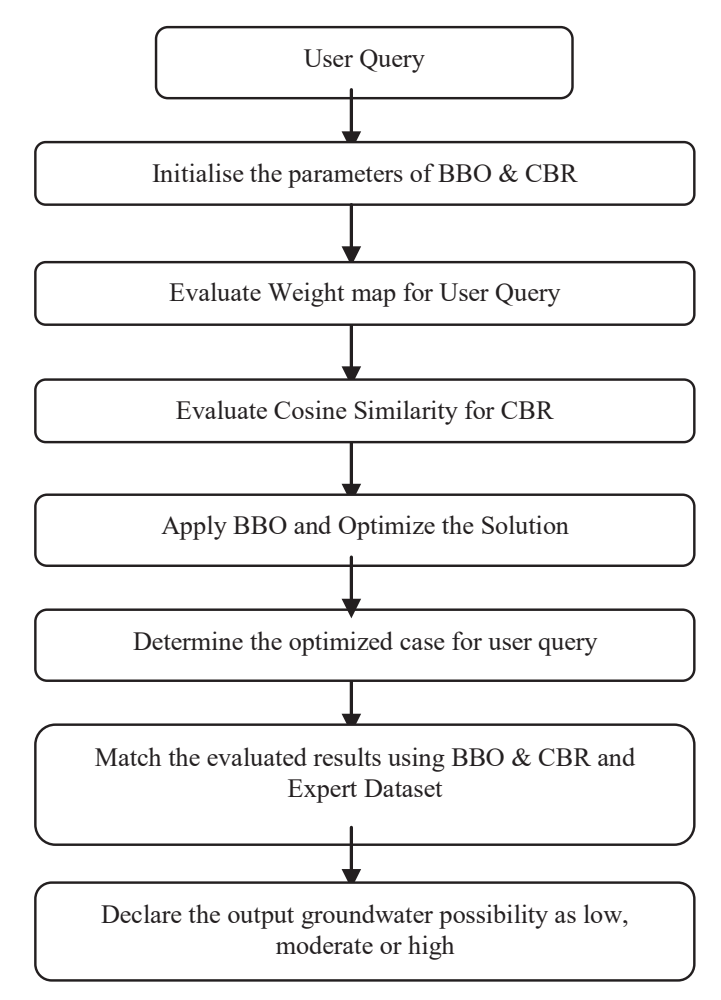

Figure 1: Flowchart for the Proposed Concept

The explanation for the work flow of proposed concept is described below.

Step 1: Consider the expert dataset and user query for the groundwater possibility detection with attributes feature values.

Step 2:Initialize the parameters of BBO like habitats, number of iterations, immigration rate and emigration rate.

Step 3:Evaluate the weight map function for the user query and use this weight map function to evaluate cosine similarity for the available use cases in CBR.

Step 4:Based on CBR values, apply BBO and optimise the solution for the groundwater possibility detection.

Step 5: Determine the optimized case with considered features of user query.

Step 6: Match the evaluated results using BBO \& CBR with the expert dataset and declare the results as low, moderate and high.

For Example: If we can consider the case of user query with the below attributes as shown in table 1,

Table 1: User Query

\begin{tabular}{|c|c|}
\hline Attribute & Attribute Value \\
\hline Lineament & Absent \\
\hline Slope & Steep \\
\hline Land Use & Urban \\
\hline Soil type & Loam \\
\hline Landform & Wadi \\
\hline Geology & Igneous \\
\hline
\end{tabular}


In this case, groundwater possibility will be low as per output results. In the similar manner, we can evaluate the results for the other queries for the groundwater possibility detection. We have evaluated the results with the number of test cases.

\section{CONCLUSIONS}

Nature is known to give optimized solutions for any of the research problem. Here, we have considered nature inspired swarm intelligence for the exploration of groundwater possibility. Groundwater is the ubiquitous source of water due to its availability in all the seasons as compare to other water resources. With the increasing usage, demand for the groundwater is also increasing. So, here we have used integrated approach of CBR and BBO for groundwater possibility detection. The proposed concept is explained with the possible example and work flow. The results for the detection shows the efficiency for the proposed concept.

\section{REFERENCES}

[1] Blum, Christian, and Xiaodong Li. "Swarm intelligence in optimization." InSwarm Intelligence, pp. 43-85. Springer Berlin Heidelberg, 2008.

[2] Garnier, Simon, Jacques Gautrais, and Guy Theraulaz. "The biological principles of swarm intelligence." Swarm Intelligence 1, no. 1 (2007): 3-31.

[3] Engelbrecht, Andries P. Fundamentals of computational swarm intelligence. John Wiley \& Sons, 2006.

[4] Bonabeau, Eric, Marco Dorigo, and Guy Theraulaz. Swarm intelligence: from natural to artificial systems. No. 1. Oxford university press, 1999.

[5] Panchal, V. K., Harish Kundra, and NavpreetKaur. "A Novel Approach to Integration of waves of swarms with case based reasoning to detect groundwater potential." In 8 th Annual Asian Conference \& Exhibition of Geospatial information technology \& application, Map Asia, Singapore. 2009

[6] Panchal, V.K., Bidisha Das, and Daya Gupta. "Applying case based reasoning in cuckoo search for the expedition of groundwater exploration." InProceedings of Seventh International Conference on Bio-Inspired Computing: Theories and Applications (BIC-TA 2012), pp. 341-353. Springer India, 2013.

[7] Gupta, Apoorva, V. K. Panchal, and Nidhi Chandra. "Robotic Perception Amalgamated with Autonomic Computing for Ground Water Level Detection." In Computational Intelligence and Communication Networks (CICN), 2014 International Conference on, pp. 12141218. IEEE, 2014.

[8] Eberhart, R. C., \& Kennedy, J. (1995, October). A new optimizer using particle swarm theory. In Proceedings of the sixth international symposium on micro machine and human science (Vol. 1, pp. 39-43).

[9] Dorigo, M., Maniezzo, V., \&Colorni, A. (1996). Ant system: optimization by a colony of cooperating agents. Systems, Man, and Cybernetics, Part B: Cybernetics, IEEE Transactions on, 26(1), 29-41.

[10] Karaboga, Dervis. An idea based on honey bee swarm for numerical optimization. Vol. 200. Technical report-tr06, Erciyesuniversity, engineering faculty, computer engineering department, 2005.

[11] Simon, Dan. "Biogeography-based optimization." IEEE transactions on evolutionary computation 12, no. 6 (2008): 702-713.

[12] Yang, Xin-She, and Suash Deb. "Cuckoo search via Lévy flights." In Nature \& Biologically Inspired Computing, 2009. NaBIC 2009. World Congress on, pp. 210-214. IEEE, 2009.

[13] Kari, Lila, and GrzegorzRozenberg. "The many facets of natural computing."Communications of the ACM 51, no. 10 (2008): $72-83$. 